\title{
Bases fisiológicas del crecimiento diferencial en la fase inicial del desarrollo de dos progenies de Pinus taeda bajo condiciones de campo, en Misiones Argentina
}

\author{
Physiological basis of differential growth in seedlings from two progenies of Pinus taeda under \\ field conditions, in Misiones Argentina
}

\author{
Nardia Bulfe $^{\text {a*}}$, Laura Faustino ${ }^{\text {b }}$, Martin Pinazo a, Corina Graciano ${ }^{\text {b }}$, María Elena Fernández ${ }^{\mathrm{c}}$ \\ * Autor de correspondencia: ${ }^{a}$ INTA EEA Montecarlo, Av. El Libertador 2472, CP: 3384, Montecarlo, Misiones, \\ Argentina, nbulfe@montecarlo.inta.gov.ar \\ ${ }^{\mathrm{b}}$ Universidad Nacional de La Plata, Instituto de Fisiología Vegetal (INFIVE), CONICET, Diag. 113 № 495, \\ CP 1900 - La Plata, Argentina. \\ ${ }^{\mathrm{c}}$ CONICET, INTA EEA Balcarce-Oficina Tandil, Tandil, Argentina.
}

\begin{abstract}
SUMMARY
The aim of this study was to evaluate morpho-physiological variables responsible for differential growth of Pinus taeda seedlings under subtropical natural environmental conditions in order to determine key eco-physiological characters for selection of better adapted genotypes in the context of climate change. The following variables were evaluated in half-sibling individuals from two mothers, a fast-growing $(\mathrm{CR})$ and a slow-growing $(\mathrm{CL})$ genotype: height and basal diameter $(\mathrm{BD})$ increment, stomatal conductance $\left(\mathrm{g}_{\mathrm{s}}\right)$, minimum and maximum daily water potential $(\Psi)$, photosynthesis at light saturation $\left(\mathrm{A}_{\mathrm{sat}}\right)$, branch specific hydraulic conductivity $\left(\mathrm{k}_{\mathrm{s}}\right)$, hydraulic conductance $\left(\mathrm{K}_{\mathrm{h}}\right)$, leaf specific hydraulic conductivity $\left(\mathrm{k}_{\mathrm{l}}\right)$ and aerial biomass allocation. The CR genotypes had higher height and $\mathrm{BD}$ increment and increased biomass production. Daily $\mathrm{g}_{\mathrm{s}}$ and $\Psi$ patterns and $\mathrm{A}_{\text {sat }}$ were similar among genotypes. In contrast, $\mathrm{CR}$ genotypes presented a higher water conduction efficiency per leaf area unit under conditions of high soil water availability, although their $\mathrm{k}_{\mathrm{s}}$ was more affected under water deficit conditions than in CL genotypes. The observed differences in biomass allocation within aerial compartments, leading to changes in the whole hydraulic architecture of the plant, would be responsible for differential growth rates. In addition, the observed trend of a lower stomatal regulation in $\mathrm{CR}$, reflected in a higher degree of anisohydrism and higher $\mathrm{k}$ losses in a dry period, suggests that these genotypes could be more vulnerable to water deficits than CL, requiring further studies to test this hypothesis.
\end{abstract}

Key words: intraspecific variation, aboveground biomass allocation, increment, plant hydraulic architecture, vapor pressure deficit.

\section{RESUMEN}

Para contribuir al conocimiento de caracteres ecofisiológicos útiles en la selección de genotipos aptos para ciertas áreas ecológicas, en el contexto de cambio climático, el objetivo del presente trabajo fue evaluar variables morfo-fisiológicas responsables del crecimiento diferencial de plantines de Pinus taeda bajo condiciones ambientales subtropicales. Se evaluaron en medio-hermanos provenientes de una madre de crecimiento rápido $(\mathrm{CR})$ y una de crecimiento lento $(\mathrm{CL})$ las variables: incremento en altura, diámetro a la altura del cuello $(\mathrm{DAC})$, conductancia estomática $\left(\mathrm{g}_{\mathrm{s}}\right)$, potencial hídrico $(\Psi)$ mínimo y máximo, fotosíntesis neta a saturación de radiación fotosintéticamente activa $\left(\mathrm{A}_{\mathrm{sat}}\right)$, conductancia $\left(\mathrm{K}_{\mathrm{h}}\right)$ y conductividad hidráulica específica $\left(\mathrm{k}_{\mathrm{s}}\right)$ y foliar específica $\left(\mathrm{k}_{\mathrm{l}}\right)$ de ramas, y asignación de biomasa aérea. Los genotipos CR presentaron mayor incremento en altura y DAC y mayor producción de biomasa aérea. Los patrones de $g_{s}, \Psi$ y A $A_{\text {sat }}$ fueron similares entre genotipos. En contraste, los genotipos CR presentaron mayor eficiencia de conducción de agua por unidad de área foliar en condiciones de alta disponibilidad de agua, aunque su $\mathrm{k}_{\mathrm{s}}$ fue más afectada bajo condiciones de déficit hídrico que en los CL. Las diferencias observadas en asignación de biomasa aérea, que modifican la arquitectura hidráulica de la planta, serían parcialmente responsables de las tasas diferenciales de crecimiento. Asimismo, la tendencia de menor regulación estomática en los genotipos $\mathrm{CR}$, reflejada en mayor grado de anisohidrismo y mayor caída en $\mathrm{k}_{\mathrm{s}}$ en períodos secos, sugiere que estos podrían ser más susceptibles al déficit hídrico que los $\mathrm{CL}$, requiriéndose más estudios para poner a prueba esta hipótesis.

Palabras clave: variación intraespecífica, asignación de biomasa aérea, incremento, arquitectura hidráulica, déficit de presión de vapor.

\section{INTRODUCCIÓN}

El proceso de cambio climático se presenta hoy como uno de los mayores desafíos para el manejo y conservación de los recursos forestales. La reducción del crecimiento y la mortalidad de árboles han sido bien documentados para diversas especies de árboles, en múltiples tipos de bosques (Allen et al. 2010). Esta mortandad de árboles se debe a 
elevado estrés fisiológico provocado por altas temperaturas y/o déficit hídrico. Si bien la mortalidad es el proceso menos deseable y más extremo de respuesta al estrés ambiental, las pérdidas de productividad son también un problema que afecta la rentabilidad, y con ello, la sustentabilidad de los sistemas forestales, principalmente aquellos basados en especies de rápido crecimiento. En este sentido, la selección genética por caracteres ecofisiológicos, tanto para aumentar los rendimientos como la eficiencia en el uso de los recursos y la resistencia a patógenos y estrés abiótico, tiene décadas de desarrollo en el área de los cultivos agronómicos. En contraste con este tipo de cultivos, menor desarrollo tiene la aplicación de criterios de selección basados en variables ecofisiológicas en el caso de especies forestales (Martin et al. 2005), aunque sus ventajas son evidentes, particularmente en el contexto del cambio climático global (IPCC 2007). Por ello, es relevante el conocimiento del conjunto de caracteres y procesos que determinan el crecimiento, el uso de los recursos y la resistencia al estrés en especies forestales de interés, de manera de contribuir al desarrollo de criterios de selección adecuados para cada caso.

Pinus taeda L., una de las principales especies utilizadas en forestaciones comerciales al sur de los Estados Unidos, fue introducida en zonas subtropicales de Sudamérica, en Brasil y Argentina, donde se verifican mayores productividades a las de su lugar de origen, alcanzando incrementos promedios anuales de entre $30-35 \mathrm{~m}^{3} \mathrm{ha}^{-1}$ año ${ }^{-1}$ (Cubbage et al. 2007). Esta alta productividad es dependiente de las condiciones edáficas del sitio (Fernández et al. 2012), así como también del mejoramiento genético que se desarrolla en la región desde la década del '70 del siglo pasado, intensificándose a fines de los '90 (Rodríguez y Gauchat 2005). Argentina cuenta con una superficie de bosques cultivados de aproximadamente un millón de hectáreas, siendo el principal polo forestal la región noreste (63\% de la superficie forestada del país). Dentro de esta región, más del $80 \%$ de las forestaciones de la provincia de Misiones corresponde al género Pinus, con preponderancia de $P$. taeda.

El informe del IPCC (2007) proyecta cambios en la temperatura y las precipitaciones para la región noreste de Argentina, previéndose eventos climáticos adversos de mayor magnitud e intensidad. En este contexto la productividad y sobrevivencia de las forestaciones estarían en situación de riesgo. Así, los procesos fisiológicos que determinan el crecimiento y las estrategias de sobrevivencia de las especies en general y de P. taeda en particular, resultan relevantes. Dentro de estas, las fases iniciales del desarrollo, período crucial para la regeneración natural y para la instalación de plantaciones comerciales, resultan particularmente de interés. Son numerosos los estudios que indican que la resistencia general al estrés es mayor en árboles grandes no senescentes que en plantines y juveniles (e.g. Niinemets 2010). La utilización de un enfoque ecofisiológico para describir y predecir la productividad se encuentra ampliamente desarrollada para $P$. taeda en su lugar de origen (e.g. Barnes 2002, Tang et al. 2003, Aspinwall et al. 2011, 2013), en el que las condiciones ambientales son diferentes a la región subtropical donde ha sido introducida. En contraste, son muy pocos los estudios ecofisiológicos de los materiales genéticos introducidos en Argentina. Una excepción son los estudios de Faustino et al. (2013), quienes aplicaron este enfoque con énfasis en respuestas a la fertilización.

Entre los principales factores que afectan la fijación de carbono en $P$. taeda se destaca la limitación estomática (Teskey et al. 1986). En algunos trabajos se ha reportado un comportamiento estacional de la $\mathrm{g}_{\mathrm{s}}$ (Tang et al. 2003, Aspinwall et al. 2011), que puede ser el resultado de la influencia de la temperatura ambiental, la humedad del aire o la intensidad e interceptación lumínica (Teskey et al. 1986, Tang et al. 2003) o la disponibilidad hídrica (Trifilò et al. 2015). La $\mathrm{g}_{\mathrm{s}}$ y la sensibilidad estomática, es decir, la respuesta de corto plazo de esta variable a condiciones del ambiente como el déficit de presión de vapor de la atmósfera (DPV), tienen además una relación estrecha con la funcionalidad del sistema conductivo (Oren et al. 1999). En el caso de P. taeda, se han observado comportamientos diferenciales en la sensibilidad estomática a la presión de vapor de la atmósfera entre genotipos (Aspinwall et al. 2011). Esto o una diferente vulnerabilidad a la cavitación del xilema podrían explicar el decrecimiento diferencial de la conductividad hidráulica específica $\left(\mathrm{k}_{\mathrm{s}}\right)$ de tallos con la disminución del agua en el suelo observado en distintos genotipos (Wang et al. 2003). Si bien la regulación estomática del intercambio gaseoso tendría influencia en el crecimiento diferencial entre genotipos, la capacidad fotosintética $\left(\mathrm{A}_{\mathrm{sat}}\right)$ no aportaría a esta diferenciación (Martin et al. 2005).

La mayoría de los trabajos que abordan la influencia de las condiciones ambientales en la fisiología y crecimiento de P. taeda se han desarrollado bajo condiciones controladas. Sin embargo, es de suma importancia sumar a este tipo de estudios conocimientos sobre el comportamiento de la especie bajo las múltiples o sucesivas condiciones de estrés que se pueden dar en situaciones de campo (Niinemets 2010). Si bien en este caso no es posible modificar dichas condiciones ni aislar factores que permitan describir relaciones causales, este tipo de estudio tiene la ventaja de someter a las plantas a una situación realista de disponibilidad de agua en el suelo, contrario a los ensayos en macetas. El presente estudio tiene como objetivo identificar bajo condiciones ambientales naturales, las variables morfo-fisiológicas responsables del crecimiento diferencial en altura y diámetro de plantines de Pinus taeda descendientes de dos genotipos seleccionados por su tasa de crecimiento contrastante. Las mediciones realizadas en condiciones ambientales pueden ser consideradas representativas del cultivo de la especie en Argentina, llevándose a cabo en un período de 15 meses con precipitación media acumulada similar a la histórica para la región, y dentro de ella con períodos contrastantes en disponibilidad hídrica, y en las 
condiciones edáficas, donde el cultivo de la especie tiene mayor desarrollo actual. Como hipótesis de trabajo se plantea que el crecimiento diferencial entre las progenies está relacionado con diferencias en comportamiento estomático, conductividad hidráulica específica del xilema y asignación de biomasa entre compartimentos aéreos (hojas vs tejido leñoso) que favorecen el aumento de la conductancia hidráulica por unidad de área foliar. En este contexto, los genotipos de rápido crecimiento presentan una menor sensibilidad estomática que los de bajo crecimiento, cerrando más tardíamente y/o en menor magnitud los estomas ante condiciones de déficit hídrico, redundando en una mayor capacidad de fijación de carbono. Esto se verá reflejado en un comportamiento más anisohídrico en los genotipos de rápido crecimiento frente a uno más isohídrico de los de lento crecimiento (según el criterio propuesto en Trifilò et al. (2015)). Asimismo, se postula que los genotipos de rápido crecimiento presentan una mayor $\mathrm{k}_{\mathrm{s}}$ de ramas o menor asignación de biomasa a hojas y una mayor a fuste que los de lento crecimiento, permitiéndoles una alta capacidad de provisión de agua por unidad de área foliar (alta conductividad hidráulica foliar específica).

\section{MÉTODOS}

En septiembre de 2009, plantines de tres meses de edad producidos de semilla proveniente de la polinización abierta (medio-hermanos) de dos genotipos de $P$. taeda clasificados como de diferentes tasa de crecimiento, fueron plantados a campo en un ensayo en el Campo Anexo Laharrague del Instituto Nacional de Tecnología Agropecuaria (INTA) de Argentina (Montecarlo, Misiones, $26^{\circ} 30^{\prime} \mathrm{S}, 54^{\circ} 40^{\prime} \mathrm{O}$ ). El clima de la región es subtropical sin estación seca marcada, con precipitación media de $2.000 \mathrm{~mm}$ anuales y temperatura media anual de $20{ }^{\circ} \mathrm{C}$. La distancia de plantación fue de $0,8 \times 0,8 \mathrm{~m}$ entre filas y líneas, minimizando la heterogeneidad espacial de las condiciones topográficas, edáficas y lumínicas. Este espaciamiento fue adecuado para la corta duración del ensayo. Se instalaron tres parcelas conteniendo la progenie de ambos genotipos dentro de cada una. Cuatro individuos de cada progenie (CR y CL, denotando crecimiento rápido y lento, respectivamente) fueron distribuidos en una línea de plantación dentro de cada parcela, teniendo 12 individuos por madre en todo el ensayo.

Las variables ambientales fueron registradas con una estación meteorológica (Davis GroWeather) situada a $100 \mathrm{~m}$ del ensayo. Debido a problemas de funcionamiento del sensor de humedad relativa que arrojó valores dudosos, el promedio del déficit de presión de vapor de la atmósfera (DPV; ecuación 1), corresponde al calculado con base en las temperaturas registradas cada 30 minutos en el sitio y período de estudio, y la humedad relativa promedio de cada mes (período 1991-2010) correspondiente a la Estación Meteorológica Iguazú (Servicio Meteorológico Nacional de Argentina). Dicha estación está situada a $120 \mathrm{~km}$ de distancia del área de estudio (figura 1).

$$
D P V(k P a)=e_{s}\left(1-\frac{H R}{100}\right)
$$

Donde $e_{s}$ corresponde a la presión de vapor saturado, estimado a través de la temperatura absoluta $\left({ }^{\circ} \mathrm{C}\right)$, y HR es la humedad relativa (\%).

El período de estudio (septiembre 2009-noviembre 2010) presentó un nivel de precipitaciones promedio casi igual a la media histórica (i.e. en promedio, llovió un $98 \%$ de la media histórica en cada mes, resultando en un valor acumulado total para los 15 meses de estudio similar al histórico). Sin embargo, se destacaron dos períodos contrastantes en cuanto a las precipitaciones: marzo, con abundantes precipitaciones ( $250 \%$ más que la histórica), y agosto como un mes de mayor estrés ambiental (sólo $13 \%$ de la precipitación histórica) (figura 1). Las condiciones de temperatura y humedad relativa en estos meses fueron no limitantes para el intercambio gaseoso debido a que no se registraron temperaturas extremas como las de verano. Las diferencias en la disponibilidad de agua en suelo en las distintas fechas fueron corroboradas por las diferencias significativas en potencial hídrico determinado en pre-alba en los individuos estudiados, considerando que la misma se encuentra en equilibrio con el suelo (ver apartado de "Resultados" con los valores medidos de esta variable). Los valores de déficit de presión de vapor de la atmósfera reportados en la figura 1 corresponden a los valores promedio mensuales, y por esta razón son inferiores a $1 \mathrm{kPa}$, valor que no refleja los picos absolutos de esta variable. En este sentido, en los registros diarios pueden observarse valores absolutos superiores a $3 \mathrm{kPa}$ en horarios de máxima temperatura (14 horas) en agosto (máxima absoluta de $31,2{ }^{\circ} \mathrm{C}$ ) y marzo (máxima absoluta de $31,5^{\circ} \mathrm{C}$ ), valores que se vieron superados durante el verano (máxima absoluta registrada en diciembre de 2009: $32,5^{\circ} \mathrm{C}$, enero 2010 : $32,7^{\circ} \mathrm{C}$, febrero $2010: 35,4^{\circ} \mathrm{C}$ ).

Los genotipos utilizados en el ensayo forman parte del Programa de Mejoramiento Genético (PMG) de esta especie llevado a cabo por el INTA. Los genotipos analizados fueron seleccionados por provenir de madres con diferentes tasas de crecimiento en volumen y rectitud de fuste, dentro de un ranking realizado al quinto año de edad. Este se elaboró mediante la combinación de ambas variables, representando cada una de ellas un $70 \%$ y $30 \%$, respectivamente. Se evaluó así la descendencia (medio-hermanos) de un genotipo catalogado como de crecimiento rápido (CR) y uno de crecimiento lento (CL).

Los registros de las variables consideradas en el ensayo comenzaron en marzo de 2010 (seis meses posteriores a la plantación), y se extendieron hasta noviembre del mismo año. Se registraron las variables altura total de cada planta $\left(\mathrm{H}_{\mathrm{t}}\right)($ precisión: $0,01 \mathrm{~m})$ con una cinta métrica y el diámetro a la altura del cuello (DAC) (precisión: 0,01 mm) con calibre digital. Con ellas se estimaron el incremento absoluto (IA) y relativo (IR) de ambas variables corres- 

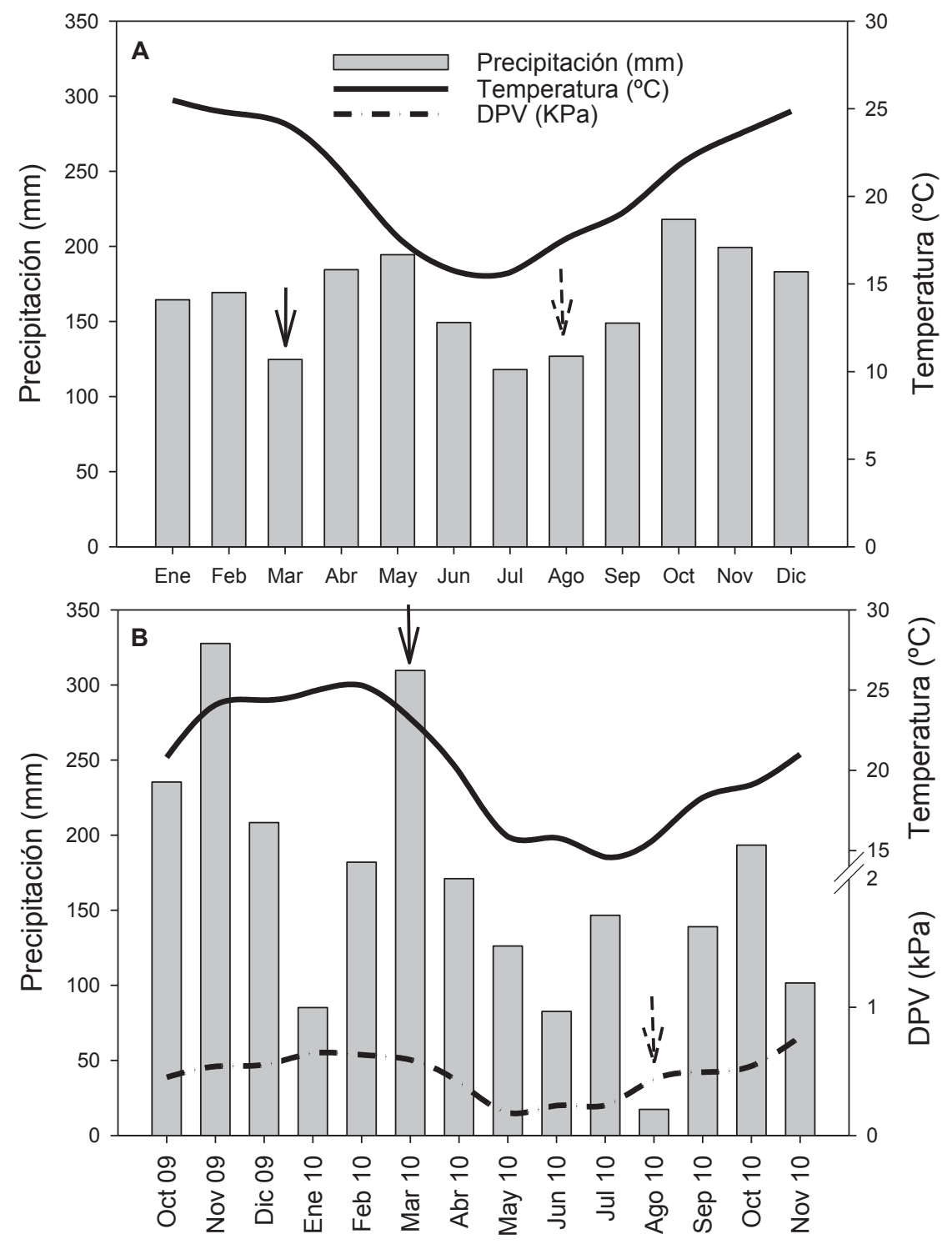

Figura 1. A) Datos climáticos de la región, correspondientes al período 1963-2010. B) Datos meteorológicos en el período primavera 2009-primavera 2010 registrados en el Campo Anexo Colonia Laharrague, Misiones, Argentina. Promedio mensual de la temperatura del aire y déficit de presión de vapor (DPV), y valor acumulado mensual de la precipitación. Flechas de línea completa corresponden a marzo (mayores precipitaciones respecto a la media climática) y flechas punteadas corresponden a agosto (menores precipitaciones respecto a la media climática).

Meteorological information of the study area. A) Climatic data of the period 1963-2010. B) Meteorological data of the period spring 2009-Spring 2010 recorded in the Campo Anexo Colonia Laharrague, Misiones, Argentina. Monthly average temperature and vapor pressure deficit (VPD), and accumulated monthly precipitation. Full line arrows indicate March values (higher rainfall compared to climate average) and dotted arrows correspond to August (less precipitation compared to the climatic average).

pondiente al período de estudio, según las ecuaciones 2 y 3 respectivamente:

$$
\begin{aligned}
& I A(m ; m m)=T_{\text {final }}-T_{\text {inicial }} \\
& I R(\%)=\frac{T_{\text {final }}-T_{\text {inicial }}}{T_{\text {inicial }}} \times 100
\end{aligned}
$$

Donde:

$\mathrm{T}_{\text {final }}=$ variable de tamaño (altura; DAC) al inicio del ensayo.

$\mathrm{T}_{\text {inicial }}=$ variable de tamaño (altura; DAC) al final del ensayo.

Se registraron las siguientes variables fisiológicas:

- Conductancia estomática $\left(\mathrm{g}_{\mathrm{s}}, \mathrm{mol} \mathrm{m}^{-2} \mathrm{~s}^{-1}\right)$ : se midió en marzo, junio y agosto de 2010 cada dos horas (10:00, 
12:00 y 14:00 h) con un porómetro Decagon Devices modelo SC-1, en dos fascículos de la porción media de la copa de dos individuos por progenie de cada parcela (seis individuos por progenie, $n=3$ ).

- Potencial hídrico en pre-alba $\left(\Psi_{\mathrm{PA}}, \mathrm{MPa}\right)$ y mediodía $\left(\Psi_{\mathrm{MD}}, \mathrm{MPa}\right)$ : se midió con una cámara de presión (Biocontrol, Argentina) en marzo y agosto de 2010 en un individuo por progenie de cada parcela $(n=3)$.

- Fotosíntesis neta a saturación de radiación fotosintéticamente activa $\left(\mathrm{A}_{\text {sat }}, \mu \mathrm{mol} \mathrm{CO} \mathrm{m}^{-2} \mathrm{~s}^{-1}\right.$, saturación lumínica a $1.500 \mu \mathrm{mol}$ fotones $\left.\mathrm{m}^{-2} \mathrm{~s}^{-1}\right)$ : se midió en marzo en tres fascículos completamente expandidos de la porción media de la copa en una planta por progenie de cada parcela $(n=3)$, con un analizador de gases infrarrojo PPSystems Ciras-2 (concentración de $\mathrm{CO}_{2}: 400$ ppm; temperatura media del aire durante la medición: $25^{\circ} \mathrm{C}$; velocidad de flujo: $300 \mu \mathrm{mol} \mathrm{s}{ }^{-1}$; tiempos de espera promedio: 10 minutos).

- Conductividad hidráulica $\left(\mathrm{k}_{\mathrm{h}}, \mathrm{kg} \mathrm{m} \mathrm{s}^{-1} \mathrm{MPa}^{-1}\right)$ de ramas, conductividad hidráulica específica $\left(\mathrm{k}_{\mathrm{s}}, \mathrm{kg} \mathrm{s}^{-1} \mathrm{~m}^{-1} \mathrm{MPa}\right.$ $\left.{ }^{1}\right)$ y foliar específica $\left(\mathrm{k}_{1}, \mathrm{~m} \mathrm{~s}^{-1} \mathrm{MPa}^{-1}\right)$ : estas variables se midieron en ramas extraídas de la parte media de la planta de tres individuos por progenie de cada parcela por el método del conductímetro multicanal (Fernández et al. 2010). La $\mathrm{k}_{1}$ se estimó como el cociente entre la $\mathrm{k}_{\mathrm{h}} \mathrm{y}$ la biomasa foliar alimentada por la rama. Al final del ensayo (en noviembre de 2010, condiciones de mayor estrés relativo) se estimó en dos individuos por progenie de cada parcela (seis individuos por progenie, $n=3$ ) la conductancia hidráulica $\left(\mathrm{K}_{\mathrm{h}}, \mathrm{kg} \mathrm{s}^{-1} \mathrm{MPa}^{-1}\right)$ en ramas con hojas, y luego, cortando la porción con hojas de cada rama, se estimó en la porción de rama la $\mathrm{k}_{\mathrm{h}} \mathrm{y}$ la $\mathrm{k}_{\mathrm{s}}$, estimándose luego la $\mathrm{k}_{1}$ a partir de la $\mathrm{k}_{\mathrm{h}} \mathrm{y}$ la biomasa foliar de la rama. Estas variables se midieron por el método de flujo estable de baja presión (SSFM, steadystate flow meter), el cual utiliza la caída de presión a través de una resistencia conocida, junto con la presión en la conexión del tallo (ver descripción del método y citas del mismo en: Faustino et al. 2013), para medir el flujo en el tallo con acículas. Las muestras fueron tomadas entre las 7:00 y 8:00 h, colocadas en bolsas plásticas y luego llevadas al laboratorio, todas con diámetros y longitudes similares.

El comportamiento estomático de las progenies se evaluó mediante la comparación de los potenciales hídricos foliares mínimos registrados en períodos secos y húmedos (Trifilò et al. 2015). Así, se considera que la especie (o progenie) posee un comportamiento isohídrico o anisohídrico si la diferencia de potencial hídrico mínimo entre situaciones es menor o mayor a $1 \mathrm{MPa}$, respectivamente.

Al finalizar el ensayo, se determinó la asignación de biomasa en los distintos compartimentos aéreos (tallo, ramas y hojas) en tres plantas por progenie de cada parcela. Se pesó el total del material en fresco de cada compartimento y luego se pesó en fresco una submuestra, que fue llevada a estufa a $65 \pm 5^{\circ} \mathrm{C}$ hasta peso constante. A partir de la relación peso fresco: peso seco de las submuestras, se estimó el peso seco de cada compartimiento (precisión: 0,01 g).

La selección de las plantas para el registro de las diferentes variables fue al azar. Todas las variables analizadas fueron evaluadas a nivel de parcela, por lo cual se consideró $n=3$, utilizando valores promedios por parcela en el caso de las variables en las que se contó con más de un dato por parcela. Los datos de incremento en altura y DAC en función del tiempo fueron analizados mediante el ajuste de un modelo exponencial, realizado con el software Table Curve 2D (Jandel Scientific, AISN Software). Los modelos completos fueron comparados entre progenies mediante la prueba $F$ (Zar 1999), con a $=0,05$. Se compararon los parámetros de los modelos entre progenies mediante la prueba $t$ de Student. Asimismo, se compararon la altura y DAC de las plantas al final del ensayo entre tratamientos mediante ANDEVA. Para el análisis de los datos de las restantes variables, se utilizó ANDEVA de una vía cuyo factor de análisis fue la progenie $(\mathrm{a}=0,05)$, aplicándose un análisis por fecha de medición cuando la variable se midió en más de una fecha. En el caso de la $\mathrm{g}_{\mathrm{s}} \mathrm{y}$ la $\mathrm{k}_{\mathrm{s}}$, además se compararon los valores dentro de cada progenie entre fechas con una prueba de $t$ de Student apareada $(\mathrm{a}=0,05)$.

\section{RESULTADOS}

Incremento en altura total y $D A C$. El incremento absoluto en altura de cada progenie entre marzo y noviembre de 2010 fue mayor $(P=0,028)$ en las plantas de crecimiento rápido $(1,09 \pm 0,17)$ que en las de crecimiento lento $(0,58$ $\pm 0,08 \mathrm{~m})$, diferenciándose estadísticamente $(P=0,028)$ (figura 2A). El incremento en DAC para el mismo período fue de $28,2 \pm 6,4$ y $15,5 \pm 2,3 \mathrm{~mm}$ para las progenies de crecimiento rápido y lento, respectivamente (figura 2B), sin diferencias significativas entre progenies $(P=0,069)$, tal vez como producto del reducido tamaño muestral ( $n$ $=3$, figura 2B). En este sentido, si se consideran las 12 plantas medidas en el análisis como muestras independientes, las diferencias en tamaño entre ellas son significativas. Los modelos exponenciales ajustados a ambas variables en función del tiempo difirieron significativamente entre progenies, tanto para la ALT $\left(\mathrm{F}_{\text {obs }}=30,697, \mathrm{~F}_{\text {crit }}=1,799\right)$ como para el DAC $\left(\mathrm{F}_{\text {obs }}=17,225 ; \mathrm{F}_{\text {crit }}=1,799\right)$. Sin embargo, considerando los parámetros individuales de los modelos, solo se observaron diferencias significativas entre progenies en el caso del parámetro $b$ del crecimiento en DAC (prueba de $t, P<0,05$ ). Los modelos exponenciales $\left(\mathrm{y}=\mathrm{a} \mathrm{e}^{\mathrm{bx}}\right)$ ajustados para la progenie de crecimiento rápido fueron: $\mathrm{ALT}=0,166 \mathrm{e}^{(0,004 \mathrm{x})}\left(\mathrm{R}^{2}\right.$ ajustado: 0,70); $\mathrm{DAC}=$ $1,890 \mathrm{e}^{(0,006 \mathrm{x})}\left(\mathrm{R}^{2}\right.$ ajustado: 0,75$)$. Para la progenie de crecimiento lento, los modelos fueron: $\mathrm{ALT}=0,114 \mathrm{e}^{(0,004 \mathrm{x})}\left(\mathrm{R}^{2}\right.$ ajustado: 0,75); $\mathrm{DAC}=2,089 \mathrm{e}^{(0,0045 \mathrm{x})}\left(\mathrm{R}^{2}\right.$ ajustado: 0,65$)$. En todos los casos, la variable independiente fue el tiempo (expresado en "cantidad de días desde la germinación", figura 2). 


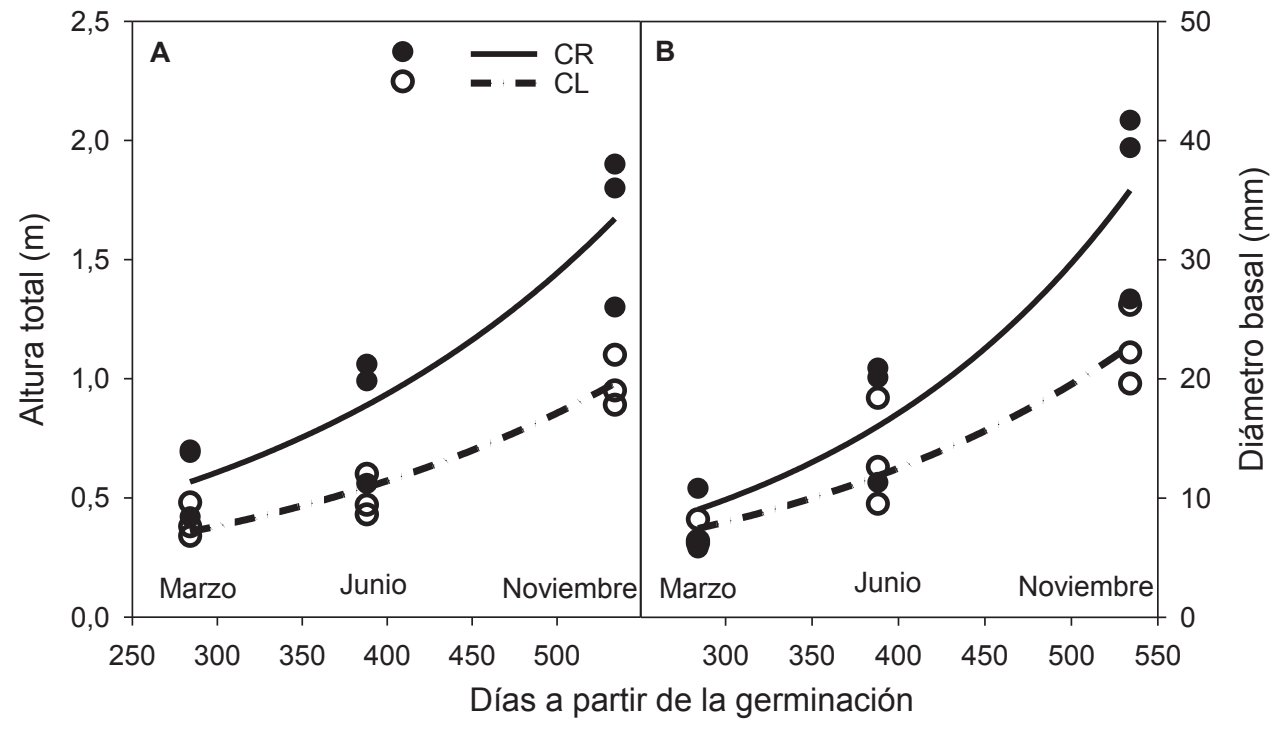

Figura 2. Evolución de: A) altura total de la planta (m) y B) diámetro basal del tallo (DAC) registrada durante el período de medición marzo-noviembre de 2010 en plantines de Pinus taeda de dos progenies del PMG del INTA. CR= progenie de crecimiento rápido, $\mathrm{CL}=$ progenie de crecimiento lento. Las líneas corresponden a los modelos exponenciales ajustados al conjunto de datos de cada progenie (ver parámetros y ajustes en el texto), los que fueron distintos entre ellas (prueba de $F, P<0,05$ ) tanto para la variable altura como para el diámetro basal. Cada punto representa el promedio de cuatro plantas medidas en cada una de las tres parcelas $(n=3)$.

Evolution of A) Total plant height (m), B) Stem basal diameter (mm) recorded between March-November, 2010, in seedlings of two progenies of Pinus taeda from de Breeding Program of INTA. $\mathrm{CR}=$ fast growth progeny, $\mathrm{CL}=$ low growth progeny. Lines indicate the exponential models applied to the data, which were different between progenies in both variables (F test, $P<0.05$; see text for models parameters). Each point represents the average of four plants in each of the three plots $(n=3)$.

Los valores promedios para el incremento relativo en altura fueron de $184,5 \pm 35,8$ y $123,3 \pm 2,9 \%$ para los genotipos de crecimiento rápido y lento, respectivamente, mientras que para la variable DAC los valores promedios fueron de 384,2 $\pm 119,9$ y 196,9 $\pm 36,2 \%$ para plantas de crecimiento rápido y lento, respectivamente.

Producción y distribución de la biomasa aérea. La biomasa aérea absoluta al final del ensayo fue significativamente mayor en la progenie de crecimiento rápido que en la de crecimiento lento (figura $3 \mathrm{~A} ; P=0,005$ ). Asimismo, la distribución relativa en los diferentes compartimentos aéreos fue diferente entre progenies (figura 3B; $P=0,01$ ). Los genotipos de crecimiento lento presentaron una mayor proporción de biomasa foliar que los de crecimiento rápido $\left(4,1 \pm 1,3\right.$ y $2,4 \pm 0,8 \mathrm{~g} \mathrm{~g}^{-1}$, respectivamente).

Conductancia estomática, potencial hídrico y fotosíntesis a saturación de RFA. Ambas progenies presentaron, dentro de cada fecha, patrones diarios similares de conductancia estomática (figura 4), con valores relativamente constantes durante todo el período evaluado ( 10 a 14 horas), sin cierre estomático en el horario de mayor demanda evaporativa (14 horas). Si bien los valores promedios de $g_{\mathrm{s}}$ no fueron diferentes entre progenies (ANDEVA, $P>0,05$ ), se observó una fuerte disminución de $\mathrm{g}_{\mathrm{s}}$ ante condiciones ambientales de mayor estrés (figura 4). La mayor $\mathrm{g}_{\mathrm{s}}$ se observó en marzo, y la menor, en agosto, cuando se registraron las menores precipitaciones. Sin embargo, dentro de cada progenie, las diferencias entre fechas fueron significativas solamente en plantas de crecimiento rápido (prueba $t$ apareada, $P<0,05)$. Los valores promedios diurnos de $\mathrm{g}_{\mathrm{s}}$ para todo el período fueron de 0,269 $\pm 0,098$ y 0,260 $\pm 0,090$ mol m $\mathrm{m}^{-2} \mathrm{~s}^{-1}$ para los genotipos de crecimiento rápido y lento, respectivamente.

Dentro de cada fecha de medición, ambas progenies presentaron similares valores de potencial hídrico en prealba y mediodía. El potencial hídrico en pre-alba descendió en agosto, denotando las menores precipitaciones en este mes que seguramente redundaron en un menor contenido de agua en el suelo (figura 5). En contraste, los menores valores de potencial al mediodía se registraron en marzo, alcanzándose valores por debajo de -4 MPa. Si bien se observó que los genotipos de crecimiento rápido alcanzaron potenciales hídricos más negativos al mediodía en ambas fechas de medición, las diferencias no fueron significativas entre progenies. Los potenciales hídricos pre-alba y mediodía manifestaron un comportamiento estacional marcado (figura 5). La diferencia de potencial entre pre-alba y mediodía, que refleja el gradiente máximo de potencial hídrico para el movimiento de agua dentro de la planta, fue máxima en marzo con una tendencia a la diferenciación entre genotipos de crecimiento rápido y lento. Las diferencias de potencial hídrico foliar mínimo 


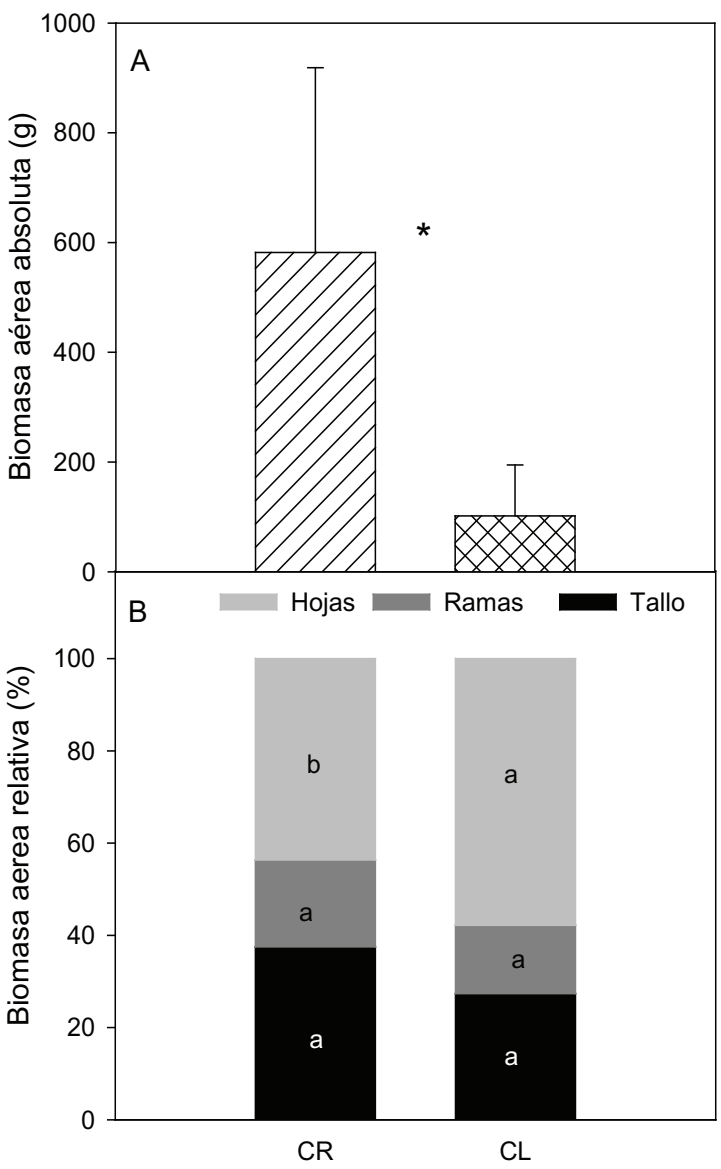

Figura 3. Biomasa aérea al final del ensayo de individuos de dos progenies de Pinus taeda. A) Biomasa aérea total del individuo (g). B) Proporción de biomasa aérea en los compartimentos tallo, ramas y hojas (\%). $\mathrm{CR}=$ progenie de crecimiento rápido; $\mathrm{CL}=$ progenie de crecimiento lento. ${ }^{*}: P<0,05$. Letras diferentes indican diferencias estadísticamente significativas (Tukey $P<0,05, n=3$ ).

Aboveground biomass at the end of the experiment in individual plants of two progenies of Pinus taeda. A) Total aboveground biomass (g), B) Distribution of aboveground biomass in stem, shoots and leaves $(\%) . \mathrm{CR}=$ fast growth progeny, $\mathrm{CL}=$ low growth progeny. $*: P<0.05$. Different letters indicate significant differences (Tukey $P<0.05, \mathrm{n}=3$ ). entre fechas con distinta disponibilidad hídrica (marzo agosto) fueron de 3,28 $\pm 1,29$ y 2,52 $\pm 1,50 \mathrm{MPa}$ para los genotipos de crecimiento rápido y lento, respectivamente (gráfico inserto dentro de la figura 5), sin diferencias entre progenies (ANDEVA, $P>0,05$ ).

La capacidad fotosintética a saturación lumínica fue similar entre progenies, con valores promedios $( \pm \mathrm{DE})$ de $18,7 \pm 2,0$ y $17,4 \pm 1,2 \mu \mathrm{mol} \mathrm{m}^{-2} \mathrm{~s}^{-1}$ para los genotipos de crecimiento rápido y lento, respectivamente.

Parámetros hidráulicos. No se observaron diferencias significativas entre progenies de crecimiento contrastante en $\mathrm{K}_{\mathrm{h}}$, cuyo promedio ( $\pm \mathrm{DE}$ ) para ambas progenies fue $5,7 \times 10^{-7} \pm 7,1 \times 10^{-7} \mathrm{~kg} \mathrm{~s}^{-1} \mathrm{MPa}^{-1}$ en noviembre de 2010. Tampoco se observaron diferencias en $\mathrm{k}_{\mathrm{h}} \mathrm{y} \mathrm{k}_{\mathrm{s}}$ de la madera entre progenies, dentro de cada fecha de medición (cuadro 1). Los promedios (ambas progenies juntas) para $\mathrm{k}_{\mathrm{h}} \mathrm{y} \mathrm{k}_{\mathrm{s}}$ en marzo fueron $3,2 \times 10^{-5} \pm 1,7 \times 10^{-5} \mathrm{~kg} \mathrm{~m} \mathrm{~s}^{-1} \mathrm{MPa}^{-1}$ y $0,57 \pm$ $0,26 \mathrm{~kg} \mathrm{~m}^{-1} \mathrm{~s}^{-1} \mathrm{MPa}^{-1}$, respectivamente. En noviembre, los promedios para las mismas variables (conjunto de ambas progenies) fueron 7,6 x 10-6 $7,9 \times 10^{-6} \mathrm{~kg} \mathrm{~m} \mathrm{~s}^{-1} \mathrm{MPa}^{-1} \mathrm{y}$ $0,47 \pm 0,67 \mathrm{~kg} \mathrm{~m}^{-1} \mathrm{~s}^{-1} \mathrm{MPa}^{-1}$. En marzo, la $\mathrm{k}_{1}$ fue similar entre progenies, aunque con una tendencia a ser mayor en plantas de crecimiento rápido que en las de crecimiento lento, al igual que lo que ocurrió en $\mathrm{k}_{\mathrm{h}} \mathrm{y}_{\mathrm{s}}$ (cuadro 1). Sin embargo, en noviembre la $\mathrm{k}_{1}$ fue significativamente inferior $(P=0,0002)$ en los genotipos de crecimiento rápido (cuadro 1).

Por otro lado, al comparar los valores de $\mathrm{k}_{\mathrm{s}}$ dentro de cada progenie en los diferentes momentos de medición, se observó una disminución significativa en los genotipos de crecimiento rápido en noviembre (mayor estrés ambiental) $(P=0,0003)$. Los genotipos de crecimiento lento presentaron valores similares para este parámetro en ambas fechas (cuadro 1).

Cuadro 1. Parámetros hidráulicos medidos en plantines de Pinus taeda de dos progenies de crecimiento contrastante. Conductividad hidráulica $\left(\mathrm{k}_{\mathrm{h}}, \mathrm{kg} \mathrm{m} \mathrm{s}^{-1} \mathrm{MPa}^{-1}\right)$, conductividad hidráulica foliar específica $\left(\mathrm{k}_{1}, \mathrm{~m} \mathrm{~s}^{-1} \mathrm{MPa}^{-1}\right)$ y conductividad hidráulica específica ( $\mathrm{k}_{\mathrm{s}}, \mathrm{kg}$ $\left.\mathrm{m}^{-1} \mathrm{~s}^{-1} \mathrm{MPa}^{-1}\right)$ de ramas medidas en marzo y noviembre de 2010 en genotipos de crecimiento rápido (CR) y crecimiento lento (CL). Las únicas diferencias significativas dentro de cada fecha se observaron en la $\mathrm{k}_{1}$ en noviembre, $P<0,05$.

Hydraulic parameters measured in Pinus taeda seedlings of two progenies with contrasting growth rate. Hydraulic conductivity ( $\mathrm{k}_{\mathrm{h}}, \mathrm{kg}$ $\left.\mathrm{m}^{-1} \mathrm{~s}^{-1} \mathrm{MPa}\right)$, specific leaf hydraulic conductivity $\left(\mathrm{k}_{1}, \mathrm{~m} \mathrm{~s}^{-1} \mathrm{MPa}^{-1}\right)$ and specific hydraulic conductivity $\left(\mathrm{k}_{\mathrm{s}}, \mathrm{kg} \mathrm{m}^{-1} \mathrm{~s}^{-1} \mathrm{MPa}^{-1}\right)$ of branches measured in March and November, 2010, in fast growth (CR) and slow growth (CL) genotypes. The only significant differences within each date were observed in November $\mathrm{k}_{1}, P<0.05$.

\begin{tabular}{ccccccc}
\hline & \multicolumn{5}{c}{ Marzo } \\
\cline { 2 - 7 } Progenies & $\begin{array}{c}\mathrm{k}_{\mathrm{h}} \\
\left(\mathrm{kg} \mathrm{m} \mathrm{s}^{-1} \mathrm{MPa}^{-1}\right)\end{array}$ & $\begin{array}{c}\mathrm{k}_{1} \\
\left(\mathrm{~m} \mathrm{~s}^{-1} \mathrm{MPa}^{-1}\right)\end{array}$ & $\begin{array}{c}\mathrm{k}_{\mathrm{s}} \\
\left(\mathrm{kg} \mathrm{m}^{-1} \mathrm{~s}^{-1} \mathrm{MPa}^{-1}\right)\end{array}$ & $\begin{array}{c}\mathrm{k}_{\mathrm{h}} \\
\left(\mathrm{kg} \mathrm{m} \mathrm{s}^{-1} \mathrm{MPa}^{-1}\right)\end{array}$ & $\begin{array}{c}\mathrm{k}_{1} \\
\left(\mathrm{~m} \mathrm{~s}^{-1} \mathrm{MPa}^{-1}\right)\end{array}$ & $\begin{array}{c}\mathrm{k}_{\mathrm{s}} \\
\left(\mathrm{kg} \mathrm{m}^{-1} \mathrm{~s}^{-1} \mathrm{MPa}^{-1}\right)\end{array}$ \\
\hline CR & $3,710^{-5} \pm 1,710^{-5}$ & $0,02 \pm 0,013$ & $0,62 \pm 0,24$ & $5,610^{-6} \pm 4,910^{-6}$ & $3,710^{-4} \pm 3,110^{-4}$ & $0,20 \pm 0,16$ \\
$\mathrm{CL}$ & $2,710^{-5} \pm 1,710^{-5}$ & $0,017 \pm 0,009$ & $0,52 \pm 0,29$ & $9,610^{-6} \pm 1,110^{-5}$ & $1,410^{-3} \pm 1,910^{-3}$ & $0,75 \pm 0,93$ \\
\hline
\end{tabular}




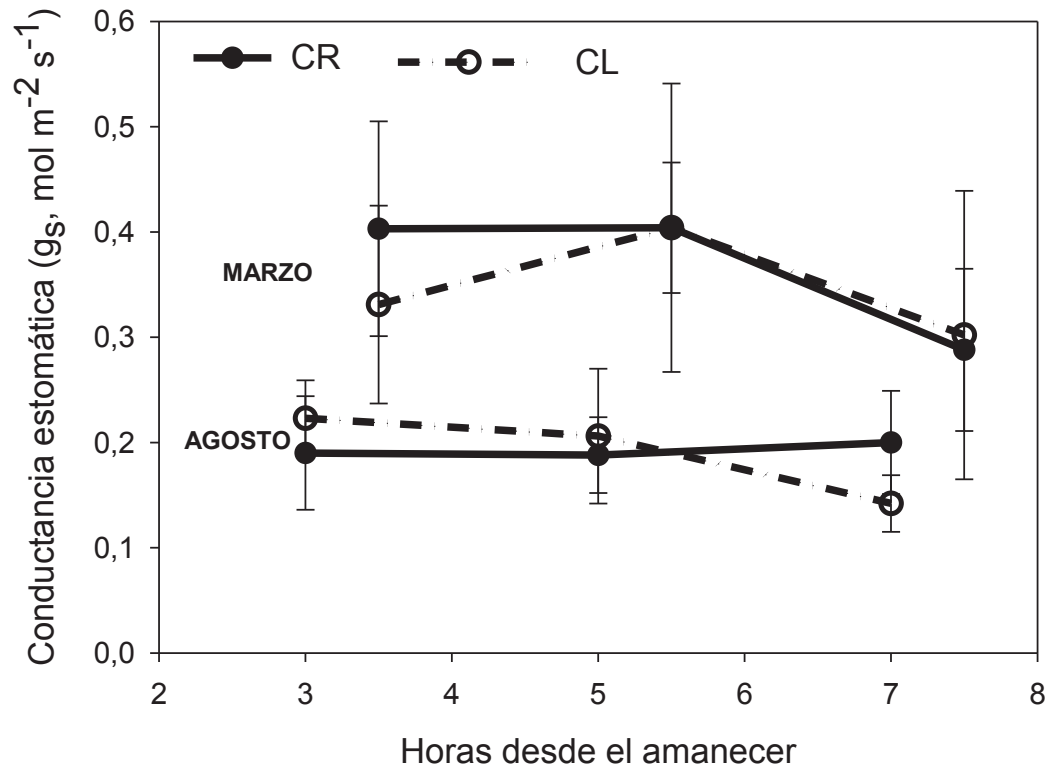

Figura 4. Evolución diurna de la conductancia estomática $\left(\mathrm{mol} \mathrm{m}^{-2} \mathrm{~s}^{-1}\right)$ promedio registrada en hojas $(n=3)$ de dos progenies de Pinus taeda en marzo y agosto de 2010. Genotipos de crecimiento rápido, $\mathrm{CR}=$ círculos y líneas completas; genotipos de crecimiento lento, $\mathrm{CL}=$ triángulos y líneas punteadas. En ninguna fecha hubo diferencias significativas entre progenies.

Diurnal pattern of average stomatal conductance $\left(\mathrm{mol} \mathrm{m}^{-2} \mathrm{~s}^{-1}\right)$ recorded in leaves $(\mathrm{n}=3)$ of two progenies of Pinus taeda in March and August, 2010. $\mathrm{CR}$ = fast growth progeny, closed circles and full lines; $\mathrm{CL}=$ low growth progeny, open circles and dashed lines. No differences were observed in either date.

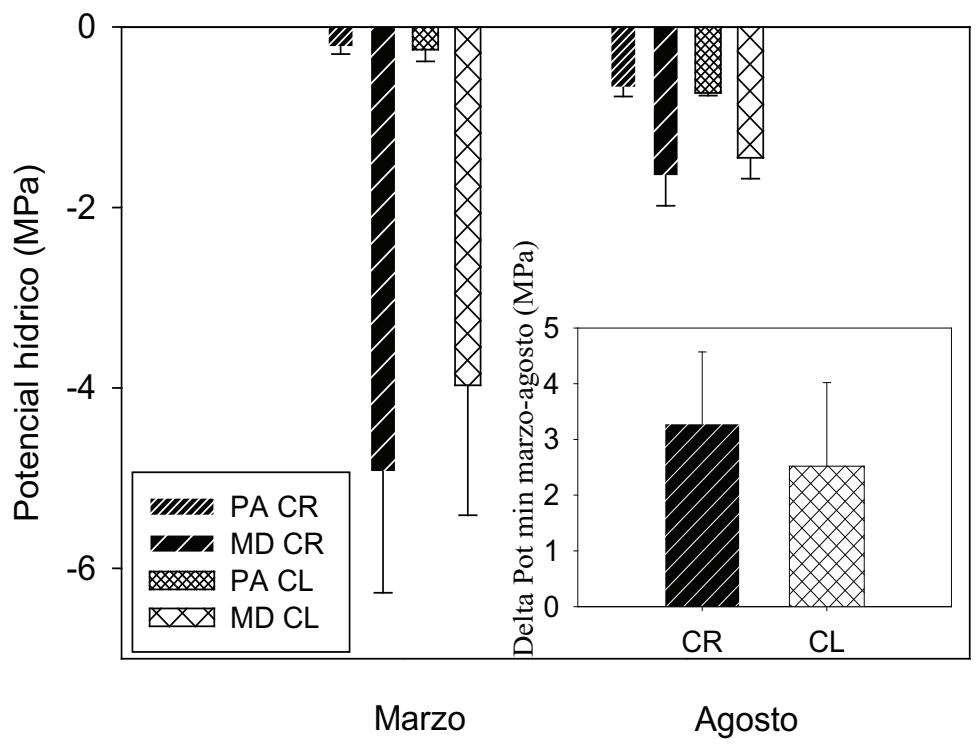

Figura 5. Estado hídrico (promedio \pm DE) en dos progenies de Pinus taeda en condiciones de campo registrado en marzo y agosto de 2010. Potencial hídrico en pre-alba (PA) y mediodía (MD), en MPa. El gráfico dentro de la figura muestra los valores promedios de las diferencias de potenciales hídricos foliares mínimos (MPa) entre períodos con alta y baja disponibilidad de agua en suelo para cada progenie. $\mathrm{CR}=$ genotipos de crecimiento rápido; $\mathrm{CL}=$ genotipos de crecimiento lento.

Water status (average \pm standard deviation) of two Pinus taeda progenies under field conditions recorded in March and August, 2010. Predawn (PA) and midday (MD) water potential, in MPa. Graph into the figure shows the difference between minimum leaf water potential (MPa) recorded in periods with high and low soil water availability. $\mathrm{CR}=$ fast growth progeny; $\mathrm{CL}=$ low growth progeny. 


\section{DISCUSIÓN}

Tal como se esperaba en función de la clasificación de sus madres, entre las dos progenies evaluadas, se han encontrado diferencias estadísticas en incrementos absolutos de altura, en la fase inicial de desarrollo de las plantas. Estos resultados coinciden con los determinados por Faustino et al. (2013) en la misma región de estudio y especie, analizando cuatro progenies con crecimientos diferenciales. El incremento relativo en altura también manifestó diferencias significativas entre progenies, no así el DAC, el cual presentó crecimiento relativo igual para ambas progenies. Las tasas diferenciales de incremento absoluto en altura observadas entre las progenies también se manifestaron en una diferenciación en la biomasa aérea al final del ensayo. Barnes (2002) encontró diferencias en biomasa total en plantines de $P$. taeda de diferentes procedencias con y sin restricción hídrica, sin embargo, los valores de biomasa determinados en el presente estudio son muy superiores (valores entre 100 y $600 \mathrm{~g}$ para biomasa aérea solamente) a los encontrados por Barnes (2002) (entre 30 y 100 g para biomasa total con y sin restricción hídrica respectivamente) en plantas de igual edad, evidenciando los crecimientos superiores alcanzados fuera de la región originaria de la especie. Los incrementos diferenciales encontrados en los genotipos implicarían a largo plazo un tamaño también diferencial, el cual se magnificaría durante el ciclo de la plantación, dadas las diferencias en cuanto a capacidades de captación de recursos entre ellos. Sin embargo, si bien los individuos de mayor tamaño podrían captar más recursos cuando estos están disponibles, estos genotipos podrían ser más sensibles ante condiciones ambientales de mayor estrés, por ejemplo, a causa de su mayor biomasa foliar absoluta (mayor superficie transpiratoria) (e.g. Niinemets 2010). Si esta mayor demanda transpiratoria no tiene un correlato en un ajustado control estomático, el xilema de estas plantas podría estar más expuesto a cavitar por las mayores tensiones. Según el criterio de Trifilò et al. (2015), queda establecido que ambas progenies presentan un comportamiento anisohídrico, dado que las diferencias del potencial hídrico mínimo entre las fechas con alta y baja disponibilidad de agua en suelo estuvieron muy por encima de $1 \mathrm{MPa}$. Sin embargo, se observó una tendencia a una mayor regulación (menor diferencia de potencial hídrico mínimo entre fechas) en los genotipos de crecimiento lento, como se hipotetizó. De acuerdo con esta hipótesis de mayor sensibilidad al estrés en los genotipos de crecimiento rápido, en el presente estudio se verificó una mayor caída en la $\mathrm{k}_{\mathrm{s}}$ de ramas en los mismos al comparar fechas, lo que sugiere una mayor vulnerabilidad a la cavitación y/o un menor control estomático del potencial hídrico. En este sentido, si bien las diferencias no fueron significativas, las plantas de crecimiento rápido tendieron a alcanzar valores de potencial hídrico más bajos que las de crecimiento lento.

Ambas progenies estudiadas no presentaron un comportamiento anisohídrico típico, debido a que los potencia- les hídricos mínimos fueron observados en el período de mayor disponibilidad de agua en suelo, contrario a lo descrito para este tipo de comportamiento estomático (Franks et al. 2007). En estos genotipos se observó una menor regulación del potencial hídrico en fechas con mayor disponibilidad de agua en suelo, aumentando la capacidad de regulación a medida que disminuye este recurso.

La distribución de la biomasa es importante desde el punto de vista funcional, y contribuye a determinar la arquitectura hidráulica de la misma. Se predice que una planta asignará más carbono a los órganos que le permitan maximizar la captura de los recursos más limitantes para su desarrollo (Barnes 2002), a la vez que los cambios en la partición a órganos que proveen agua vs los que transpiran, determinan, en parte, la eficiencia hidráulica del sistema en su conjunto. Su distribución en distintos compartimentos podría ser considerada una variable de interés en la selección temprana de genotipos. La variación intraespecífica en la partición de la biomasa en P. taeda ha sido reportada tanto en condiciones de campo (Aspinwall et al. 2013) como bajo invernáculo, donde fueron más marcadas las diferencias ante condiciones de estrés hídrico (Barnes 2002). En árboles de mayor tamaño de $P$. taeda (15 años de edad) que los estudiados aquí, Blazier et al. (2004) observaron que distintos genotipos presentaron diferentes asignaciones a hojas y ramas, e iguales proporciones de biomasa de tallo entre ellos, coincidiendo con nuestros resultados. Sin embargo, Aspinwall et al. (2013) mencionan que, si bien la partición de la biomasa representa importantes implicancias para la captura y fijación del carbono en diferentes genotipos, las diferencias intraespecíficas en productividad de $P$. taeda no pueden ser explicadas solamente por los patrones diferenciales en esta variable.

Por otro lado, en general no se encontraron diferencias significativas en la fisiología foliar entre progenies durante el período de medición, coincidiendo con Blazier et al. (2004), quienes reportaron la falta de diferencias entre progenies de P. taeda para las variables $\mathrm{g}_{\mathrm{s}}, \mathrm{A}_{\mathrm{sat}} \mathrm{y}$ potencial hídrico foliar durante la estación de crecimiento. El hecho de que ambas progenies hayan presentado similar capacidad fotosintética $\left(\mathrm{A}_{\text {sat }}\right)$ y que los genotipos de crecimiento lento asignen más biomasa a hojas, sugiere que estos podrían estar fijando igual o más carbono que los de crecimiento rápido, dependiendo de los valores absolutos de biomasa foliar alcanzados en cada caso, y sin tomar en cuenta las posibles limitaciones hidráulicas (ver próximo párrafo). El menor crecimiento aéreo observado en los genotipos de crecimiento lento sugiere entonces que tendrían una mayor asignación de fotoasimilados a las raíces, lo que concuerda con mediciones realizadas en ensayos en macetas con estas mismas progenies, que mostraron mayor asignación a raíces en los genotipos de crecimiento lento (Bulfe y Fernández 2016). Por otro lado, un comportamiento estacional para estas variables fisiológicas ha sido reportado para la especie por varios autores (Tang et al. 2003, Blazier et al. 2004, Aspinwall et al. 2011), coincidiendo con los resultados del presente estudio, 
afectando de igual manera ambas progenies. Sin embargo, la caída relativa en $\mathrm{g}_{\mathrm{s}}$ fue significativamente mayor en los genotipos de crecimiento rápido que en los de crecimiento lento al comparar fechas de medición (si bien dentro de cada fecha, no hubo diferencias significativas entre progenies), lo que sugiere que los genotipos de crecimiento rápido tendrían mayores limitaciones hídricas en condiciones adversas, posiblemente dadas por su mayor tamaño total. Por otro lado, dentro de cada fecha, y al analizar el comportamiento estomático y los valores de potencial hídrico mínimo alcanzados, se observó en ambas progenies un bajo control estomático del potencial, principalmente en los genotipos de crecimiento rápido. En este sentido, si bien se encontró evidencia preliminar a favor de la hipótesis planteada de que los genotipos de crecimiento rápido tendrían menor sensibilidad estomática, no se encontraron diferencias significativas. Sin embargo, las tendencias observadas en los parámetros que hacen referencia al comportamiento estomático sugieren que esta hipótesis podría ser comprobada, por lo cual se debería ampliar el tamaño de la muestra a futuro.

En general la conductividad hidráulica específica $\left(\mathrm{k}_{\mathrm{s}}\right)$ de distintos órganos, como las ramas, está relacionada en forma directa con el crecimiento de los individuos (e.g. Domec y Gartner 2003, Wang et al. 2003, Gonzalez-Benecke y Martin 2010). Contrariamente, la $\mathrm{k}_{\mathrm{s}}$ medida en ramas en este estudio no presentó diferencias significativas entre progenies bajo condiciones ambientales favorables. La ausencia de diferencias pudo deberse al relativamente bajo $n$ utilizado, aunque estudios complementarios al presente (Bulfe y Fernández 2016) son igualmente conducentes a afirmar que esta variable no tiene correlación con el crecimiento diferencial entre progenies. Por otro lado, Wang et al. (2003) determinaron diferencias en $\mathrm{k}_{1}$ entre progenies de $P$. contorta, variable que presentó una relación directa con la tasa de crecimiento de dicha especie. En el presente estudio, la menor asignación relativa de biomasa a hojas en los genotipos de crecimiento rápido contribuyó en el mismo sentido, es decir, se observó una tendencia a una mayor $\mathrm{k}_{1}$ en estos genotipos en marzo, período con menor estrés ambiental, aunque las diferencias no llegaron a ser significativas debido a la alta dispersión de los valores de la $\mathrm{k}_{\mathrm{h}}$, componente del cálculo de $\mathrm{k}_{\mathrm{l}}$. En este sentido, más allá de estos resultados de $\mathrm{k}_{1}$ provenientes de un único momento del día, la menor (y significativa) proporción de biomasa foliar alimentada por cada rama en los genotipos de crecimiento rápido, seguramente, tiene un alto impacto en la $k_{1}$ de manera sostenida en el tiempo. De acuerdo con la hipótesis inicialmente planteada, esto permitiría posiblemente una mayor eficiencia de conducción de agua por unidad de área foliar en los genotipos de crecimiento rápido cuando el agua en suelo no es limitante. De esta manera podrían alcanzar un incremento relativo similar (o absoluto mayor) a pesar de una menor inversión en superficie de captación de radiación y fijación de carbono, respecto a los genotipos de crecimiento lento. Cuando el agua en suelo se torna limitante (noviembre en este estudio), la $\mathrm{k}_{1}$ cae significativamente en los genotipos de crecimiento rápido como resultado de la disminución en la $\mathrm{k}_{\mathrm{h}}$, alcanzando valores por debajo de los genotipos de crecimiento lento. El impacto de esta caída en la eficiencia de conducción de agua en el crecimiento global y supervivencia de las plantas dependerá posiblemente de la duración e intensidad del estrés y de la capacidad de recuperación de la conductividad hidráulica del xilema (resiliencia) de estos genotipos.

Tomando como base los resultados aquí presentados, se puede inferir que ambas progenies tienen diferentes capacidades de crecimiento (absoluto y relativo) en altura desde muy tempranamente en su desarrollo. En contraste, las diferencias en crecimiento en DAC no son tan marcadas. Asimismo, los genotipos de crecimiento rápido acumulan mayor biomasa aérea a edades tempranas, coincidiendo con la fase de establecimiento de una plantación. Estas diferencias de tamaño manifestadas a temprana edad podrían deberse a una susceptibilidad diferencial al estrés durante el establecimiento y/o a una distinta asignación inicial de biomasa entre órganos aéreos y subterráneos. Barnes (2002) ha propuesto que en la fase de establecimiento (primera etapa) ocurre un rápido crecimiento subterráneo a expensas de biomasa foliar, siguiéndole una etapa de desarrollo foliar a expensas de las raíces (fase de crecimiento en altura, posterior al establecimiento). Si bien aún resta determinar si entre los genotipos de crecimiento rápido y lento podría existir solo un desfasaje temporal en el pasaje de una etapa a la otra, ensayos en macetas (Bulfe y Fernández 2016) muestran que los genotipos de crecimiento lento poseen una mayor asignación a biomasa radical durante el primer año de desarrollo de las plantas. Un mayor desarrollo relativo de la radícula (y posteriormente, la raíz) en los genotipos de crecimiento lento sería consistente con una mayor conductancia hidráulica total resultante en la tendencia (no significativa) observada a desarrollar potenciales hídricos mayores, a iguales $g_{s}$ y demanda evaporativa, en estos genotipos en comparación con los de crecimiento rápido a pesar de su mayor superficie transpiratoria (en relación a la biomasa de ramas) y menor $\mathrm{k}_{1}$ de ramas.

\section{CONCLUSIONES}

Coincidiendo con los criterios de selección genética de los árboles madre, la descendencia del genotipo clasificado como de rápido crecimiento presenta incrementos en altura superiores, así como mayor producción de biomasa aérea al final del período de ensayo. Tal como se hipotetizó, la progenie de crecimiento rápido se diferencia de la de crecimiento lento por una menor asignación de biomasa a hojas y una mayor asignación relativa a ramas, redundando en una mayor eficiencia de conducción de agua, en situaciones de alta disponibilidad de agua en suelo, a pesar de presentar similares $\mathrm{k}_{\mathrm{s}}$ que los genotipos de crecimiento lento. Así, los cambios observados en la asignación del carbono dentro de los distintos compartimientos de la parte aérea de la planta, que conducen a cambios en la arquitec- 
tura hidráulica de la misma, podrían ser responsables -al menos en parte- de las tasas diferenciales de crecimiento entre los genotipos de $P$. taeda estudiados.

Por otro lado, los genotipos de crecimiento rápido presentan una mayor pérdida relativa de $\mathrm{k}_{\mathrm{s}}$ ante condiciones de estrés (período de bajas precipitaciones) que los genotipos de crecimiento lento, así como una mayor caída relativa en $\mathrm{g}_{\mathrm{s}}$. Sin embargo, estas diferencias no fueron de una magnitud tal que permitiera detectar diferencias estadísticas entre progenies en variables funcionales como potencial hídrico en pre-alba y mediodía, $\mathrm{g}_{\mathrm{s}} \mathrm{y}_{\mathrm{sat}}$ dentro de cada una de las fechas de medición. Esto sugiere la necesidad de aumentar el tamaño muestral y las fechas de medición a fin de confirmar la ausencia de diferencias en estas variables, o bien confirmar algunas tendencias observadas, como el menor potencial hídrico mínimo en los genotipos de crecimiento rápido. Ambas progenies manifestaron un comportamiento estomático anisohídrico, con tendencia a una mayor regulación del potencial (menor diferencia entre fechas) en los genotipos de crecimiento lento que en los de crecimiento rápido. De confirmarse esta tendencia con un mayor número de mediciones, esta podría ser una variable más que explique el crecimiento diferencial entre progenies debido a que los genotipos de crecimiento rápido tendrían una mayor fijación de carbono (por menor cierre estomático) en los períodos de alta disponibilidad de agua en el suelo, que es cuando se verificaron los potenciales hídricos más bajos. Dado que los caracteres morfofisiológicos presentan patrones diferentes en la ontogenia de un individuo, estos resultados son exclusivos para la fase inicial de establecimiento de la plantación.

Finalmente, los resultados sugieren que los genotipos de crecimiento rápido posiblemente sean más eficientes en el uso de los recursos del ambiente, por poseer menor (en términos relativos a su biomasa) superficie transpiratoria. Se requieren estudios de patrones de enraizamiento diferencial entre genotipos en condiciones de campo, así como de uso de los recursos (agua, nitrógeno, radiación) y productividad para poner a prueba estas hipótesis. Asimismo, algunas tendencias observadas sugieren que estudios futuros debieran enfocarse en las implicancias de las tasas diferenciales de crecimiento $-\mathrm{y}$ los procesos fisiológicos que las determinan- sobre la sensibilidad y resiliencia de las distintas progenies ante condiciones de estrés ambiental.

\section{AGRADECIMIENTOS}

Los autores agradecen al INTA por el financiamiento del proyecto, en el cual fue desarrollado el presente trabajo. Al Ing. Aldo Keller por su colaboración en los trabajos de campo.

\section{REFERENCIAS}

Allen CD, AK Macalady, H Chenchouni, D Bachelet, N McDowell, M Vennetier, T Kitzberger, A Rigling, DD Bres- hearsi, EH Hogg, P Gonzalez, R Fensham, Z Zhang, J Castro, N Demidova, JH Lim, G Allard, SW Running, A Semerci, N Cobb. 2010. A global overview of drought and heat-induced tree mortality reveals emerging climate change risks for forests. Forest Ecology and Management 259(4): 660-684.

Aráos S, O Del Longo, O Karlin. 2004. Germinación de semillas de Zizyphus mistol Grisebach III. Correlaciones paramétricas del tamaño y peso de drupas, endocarpos y semillas con la germinación y el vigor. Multequina 13(1): 51-56.

Aspinwall MJ, JS King, SE McKeand, JC Domec. 2011. Leaflevel gas exchange uniformity and photosynthetic capacity among loblolly pine (Pinus taeda L.) genotypes of contrasting inherent genetic variation. Tree Physiology 31(1): 7891.

Aspinwall MJ, JS King, SE McKeand. 2013. Productivity differences among loblolly pine genotypes are independent of individual-tree biomass partitioning and growth efficiency. Trees 27(3): 533-545.

Barnes AD. 2002. Effects of phenology, water availability and seed source on loblolly pine biomass partitioning and transpiration. Tree Physiology 22(10): 733-740.

Blazier MA, TC Hennessey, TB Lynch, RF Wittwer, ME Payton. 2004. Productivity, crown architecture, and gas exchange of North Carolina and Oklahoma/Arkansas loblolly pine families growing on a droughty site in southeastern Oklahoma. Forest Ecology and Management 194(1-3): 83-94.

Bulfe NML, ME Fernández. 2016. Morpho-physiological response to drought of progenies of Pinus taeda L. contrasting in mean growth rate. New Forests 47(3): 431-451. DOI: 10.1007/s11056-016-9524-x

Cubbage F, P Mac Donagh, J Sawinski, R Rubilar, P Donoso, A Ferreira, V Hoeflich, V Morales Olmos, G Ferreira, G Balmelli, J Siry, MN Báez, J Alvarez. 2007. Timber investment returns for selected plantations and native forests in South America and the Southern United States. New Forests 33(3): 237-255.

Domec JC, BL Gartner. 2003. Relationship between growth rates and xylem hydraulic characteristics in young, mature and old-growth ponderosa pine trees. Plant, Cell and Environment 26(3): 471-483.

Faustino LI, NML Bulfe, MA Pinazo, SE Monteoliva, C Graciano. 2013. Dry partitioning and hydraulic traits in Young Pinus taeda trees fertilized with noitrogen and phosphorus in a subtropical area. Tree Physiology 33(3): 241-251.

Fernández ME, J Gyenge, C Graciano, S Varela, G Dalla Salda. 2010. Conductancia y conductividad hidráulica. In ME Fernández, J Gyenge eds. Técnicas de medición en Ecofisiología vegetal. Concepto y procedimientos. Buenos Aires, Argentina. INTA. p. 53-68.

Fernández RA, AM Lupi, R Pezutti, R Martiarena, N Pahr, A Von Wallis. 2012. Respuesta del crecimiento de Pinus taeda y Pinus elliotti a los 10 años de edad a técnicas de establecimiento en suelos hidromórficos del noreste de Argentina. In $15^{\text {as }}$ Jornadas Técnicas Forestales y Ambientales. 7 al 9 de junio de 2012. Misiones, Argentina. Facultad de Ciencias Forestales, UNaM - EEA Montecarlo, INTA. 7 p.

Franks PJ, PL Drake, RH Froend. 2007. Anisohydric but isohydrodynamic: seasonally constant plant water potential gradient explained by a stomatal control mechanism incorporating variable plant hydraulic conductance. Plant, Cell and 
Environment 30(1): 19-30.

Gonzalez-Benecke CA, TA Martin. 2010. Water availability and genetic effects on water relations of loblolly pine (Pinus taeda) stands. Tree Physiology 30(3): 376-392.

IPCC (Intergovernmental Panel on Climate Change, CH). 2007. Cambio climático 2007: Informe de síntesis. Cuarto Informe de evaluación del Grupo Intergubernamental de Expertos sobre el Cambio Climático. Ginebra, Suiza. IPCC. 104 p.

Martin TA, PM Dougherty, MA Topa, SE McKeand. 2005. Strategies and case studies for incorporating ecophysiology into southern pine tree improvement programs. Southern Journal of Applied Forestry 29(2): 70-79.

Niinemets U. 2010. Responses of forest trees to single and multiple environmental stresses from seedlings to mature plants: Past stress history, stress interactions, tolerance and acclimation. Forest Ecology and Management 260(10): 16231639.

Oren R, JS Sperry, GG Katul, DE Pataki, BE Ewers, N Phillips, KVR Schäfer. 1999. Survey and synthesis of intra- and interspecific variation in stomatal sensitivity to vapour pressure deficit. Plant Cell and Environment 22(12):1515-1526.

Premoli A. 1991. Morfología y capacidad germinativa en poblaciones de Nothofagus antartica (Forster) Oerst. Del noroeste andino patagónico. Bosque 12(1): 53-59.

Rodríguez G, ME Gauchat. 2005. Subprogramas de producción de material de propagación mejorado. Mejora genética. In R Fernández ed. Mejores árboles para más forestadores: el programa de producción de material de propagación mejorado y el mejoramiento genético en el Proyecto Forestal de Desarrollo. Buenos Aires, Argentina. Secretaria de Agricultura, Ganadería, Pesca y Alimentos. p. 23-41.

Tang Z, JL Chambers, MA Sword, JP Barnett. 2003. Seasonal photosynthesis and water relations of juvenile loblolly pine relative to stand density and canopy position. Trees $17(5)$ : 424-430.

Teskey R0, JA Fites, LJ Samuelson, BC Bongarten. 1986. Stomatal and nonstomatal limitations to net photosynthesis in Pinus taeda L. under different environmental conditions. Tree Physiology 2(1-2-3): 131-142.

Trifilò P, A Nardini, MA Lo Gullo, PM Barbera, T Savi, F Raimondo. 2015. Diurnal changes in embolism rate in nine dry forest trees: relationships with species-specific xylem vulnerability, hydraulic strategy and wood traits. Tree Physiology 35(7): 694-705.

Wang T, SN Aitken, KL Kavanagh. 2003. Selection for improved growth and wood quality in loblolly pine: effects on phenology, hydraulic architecture and growth of seedlings. Trees 17(3): 269-277.

Zar JH. 1999. Biostadistical Analysis. New Jersey, USA. Prentice Hall Press. 663 p. 